\title{
Assessment of pharmacokinetics for microvessel proliferation by DCE-MRI for early detection of physeal bone bridge formation in an animal model
}

\author{
Bernhard Neumayer ${ }^{1,2} \cdot$ Eva Amerstorfer $^{3} \cdot$ Clemens Diwoky $^{2,4} \cdot$ Richard A. Lindtner $^{5} \cdot$ Elisabeth Wadl $^{6}$. \\ Eva Scheurer $^{7} \cdot$ Annelie-Martina Weinberg $^{8} \cdot$ Rudolf Stollberger $^{2,9}$
}

Received: 19 October 2016 / Revised: 26 February 2017 / Accepted: 7 March 2017 / Published online: 30 March 2017

(C) The Author(s) 2017. This article is an open access publication

\begin{abstract}
Objectives Bone bridge formation occurs after physeal lesions and can lead to growth arrest if not reversed. Previous investigations on the underlying mechanisms of this formation used histological methods. Therefore, this study aimed to apply a minimally invasive method using dynamic contrast-enhanced MRI (DCE-MRI).

Materials and methods Changes in functional parameters related to the microvessel system were assessed in a longitudinal study of a cohort of an animal model applying a reference region model. The development of morphology of the injured physis was investigated with 3D highresolution MRI. To acquire complementary information for
\end{abstract}

Rudolf Stollberger

rudolf.stollberger@tugraz.at

1 Ludwig Boltzmann Institute for Clinical Forensic Imaging, Universitätsplatz 4, $8010 \mathrm{Graz}$, Austria

2 BioTechMed, University of Graz, Universitaetsplatz 3, 8010 Graz, Austria

3 Department of Paediatric and Adolescent Surgery, Medical University of Graz, Auenbruggerplatz 34, 8036 Graz, Austria

4 Institute of Molecular Biosciences, University of Graz, Humboldtstraße 50, 8010 Graz, Austria

5 Department of Trauma Surgery, Medical University of Innsbruck, Anichstrasse 35, 6020 Innsbruck, Austria

6 Department of Pathology, Clinical Center Klagenfurt, Feschnigstraße 11, 9020 Klagenfurt, Austria

7 Institute of Forensic Medicine, University of Basel, Pestalozzistraße 22, 4056 Basel, Switzerland

8 Department of Orthopedics and Orthopedic Surgery, Medical University of Graz, Auenbruggerplatz 5, 8036 Graz, Austria

9 Institute of Medical Engineering, Graz University of Technology, Stremayrgasse 16/III, 8010 Graz, Austria
MRI-related findings qRT-PCR and immunohistochemical data were acquired for a second cohort of the animal model.

Results The evaluation of the pharmacokinetic parameters showed a first rise of the transfer coefficient 7 days postlesion and a maximum 42 days after operation. The analysis of the complementary data showed a connection of the first rise to microvessel proliferation while the maximum value was linked to bone remodeling.

Conclusion The pharmacokinetic analysis of DCE-MRI provides information on a proliferation of microvessels during the healing process as a sign for bone bridge formation. Thereby, DCE-MRI could identify details, which up to now required analyses of highly invasive methods.

Keywords Contrast agents $\cdot$ Animal model $\cdot$ Physis

$\begin{array}{ll}\text { Abbreviations } \\ K^{\text {trans }} & \text { Transfer coefficient } \\ v_{\mathrm{e}} & \text { Extravascular extracellular volume } \\ \text { AIF } & \text { Arterial input function } \\ \text { CA } & \text { Contrast agent } \\ \text { DCE } & \text { Dynamic contrast enhanced } \\ \text { FA } & \text { Flip angle } \\ \text { FLASH } & \text { Fast low angle shot } \\ \text { FOV } & \text { Field of view } \\ \text { Hifla } & \text { Hypoxia-inducible factor 1a } \\ \text { PD } & \text { Proton density } \\ \text { RARE } & \text { Rapid acquisition with relaxation enhancement } \\ \text { RNA } & \text { Ribonucleic acid } \\ \text { RR(M) } & \text { Reference region (model) } \\ \text { SNR } & \text { Signal-to-noise ratio } \\ \text { TOI } & \text { Tissue of interest } \\ \text { VEGFa } & \text { Vascular endothelial growth factor a }\end{array}$

Abbreviations

$v_{\mathrm{e}} \quad$ Extravascular extracellular volume

AIF Arterial input function

CA Contrast agent

DCE Dynamic contrast enhanced

FA Flip angle

FLASH Fast low angle shot

FOV Field of view

Hif1a Hypoxia-inducible factor 1a

PD Proton density

RARE Rapid acquisition with relaxation enhancement

RNA Ribonucleic acid

$\mathrm{RR}(\mathrm{M}) \quad$ Reference region (model)

SNR Signal-to-noise ratio

VEGFa Vascular endothelial growth factor a 


\section{Introduction}

Premature physeal bone bridge formation can be induced by a variety of physeal insults including trauma, infection, radiation, ischemia, thermal injury, Blount disease, steroid use, as well as, iatrogenic and unknown etiologies [1]. Trauma in general including iatrogenic trauma from metal implantation is considered as the leading cause for formation of a physeal bone bridge [2, 3]. Bone bridges may be reversible when they are small, but can also trigger full or partial premature physeal closure due to incomplete decomposition and therefore may lead to full or partial growth arrest causing bone length discrepancy, axis deviation or joint deformity [4-7]. Clinically, they are detected by X-ray examinations, which are either routinely conducted to document fracture healing or initiated in patients with posttraumatic bone length alterations or deformities. MRI is then used to document the full size of the bridge and help in defining further management $[1,4,8,9]$.

A severe physeal bone bridge alters normal longitudinal bone growth and requires either surgical resection and filling up the lesion with autologous fat tissue as remedial measure or it is managed by contralateral epiphysiodesis or corrective osteotomy [1, 5, 6, 9]. Furthermore, stem cell therapy [10] and autogenous cultured physeal chondrocyte transplantation [11] have been recently investigated experimentally as novel therapeutic option. In contrast, minor bone bridges usually remain concealed and disappear during further growth, without impairing normal longitudinal growth $[6,7,12]$. Physeal bone bridge formation has been documented histologically [13-18] and by MRI [15, $16,18]$ in various experimental animal models. Vascularization of the physeal lesion has been reported to precede physeal bone bridge formation $[15,19]$. Along with invading vessels from surrounding epiphyseal and metaphyseal bone, mesenchymal osteoprogenitor cells emerge into the physeal lesion site and are attributed to deposit bone which eventually leads to bone bridge formation [15, 19]. The hypothesis that increased blood supply at the injury site is connected to the creation of physeal bone bridges as blood vessels and vascular invasion are fundamental for the development of bone tissue was further recently supported by the detection of increased angiogenic factor expression within the physeal injury site prior to bone bridge development $[13,20]$. However, the underlying pathomechanism inducing angiogenesis followed by formation of a physeal bone bridge remains unexplored. The detection of an increase in blood supply in a physeal lesion could therefore allow an early identification of bone bridge formation and while the determination of angiogenic expression factors requires tissue samples, magnetic resonance imaging is potentially capable of providing this information non-invasively.
Dynamic contrast enhanced MRI (DCE-MRI) is sensitive to functional parameters of the microvessel system. It is therefore a tool to characterize angiogenesis and microvasculature and is widely used for the analysis of tumors [21-23]. The identification of pharmacokinetic parameters should therefore allow following the time course of microvessel-related functional changes during tissue remodeling on an injured physis.

Typical models for the identification of pharmacokinetic parameters [24] require information on the time course of tissue contrast agent (CA) concentration and on the CA concentration within a feeding artery-the arterial input function (AIF). The simultaneous acquisition of both characteristics, i.e. the coverage of the AIF and, in this case, the physeal lesion in the same field of view (FOV), is very challenging and determines the limits for signal-to-noise ratio (SNR), contrast behavior and temporal or spatial resolution [25-27].

For investigations where no adequate arterial input function is available in the field of view populationbased averaged AIFs [28-30] or model-based arterial input functions [31] can be applied to avoid a decrease in SNR or temporal resolution by modifying the FOV. A drawback of these methods is, however, that interindividual variations between subjects or variations of the AIF due to manual CA administration cannot be covered. A different approach is to determine the CA dynamics of a reference tissue with known pharmacokinetic behaviour within the field of view. These dynamic data can then be used to apply a reference region model (RRM) which allows for the quantification of the pharmacokinetic parameters of a tissue of interest without the direct measurement of or assumptions on the AIF $[32,33]$.

The aim of this study was to investigate pharmacokinetic parameters of the microvessel system of a physeal lesion in correlation to bone bridge formation. These parameters are typically increased in tissues with enhanced metabolism and related to permeability surface product, blood flow and also blood volume. The investigation of these parameters should support the hypothesis that changes of the microvessel system are basic mechanisms associated with bone bridge formation and can provide quantitative measures for an early identification of this development.

The investigation of physeal lesions in a rat model using DCE-MRI required a very high spatial resolution and the application of a reference region model. As a complementary independent measure the results of the MRI analyses were compared to histologic findings and expression rates of genes related to vascular growth and oxygenation provided by quantitative real-time reverse transcription 
polymerase chain reaction data (qRT-PCR), which were acquired for a second cohort of animals.

\section{Materials and methods}

\section{Animals}

All animal experiments were conducted under animal ethical respect and were authorized by the Austrian Ministry of Science and Research. Seventy-seven male Sprague-Dawley rats with a weight of approximately $100 \mathrm{~g}$ and an age of one month were subjected to unilateral physeal lesion in general anesthesia using a standardized drill procedure $[13,14,17,34]$. A longitudinal transepiphyseal lesion of $1.2 \mathrm{~mm}$ diameter was drilled through the proximal tibial physis, advanced from proximal by a median incision of the patella tendon.

For qRT-PCR analyses six animals were euthanized on days $0,1,3,7,14,28$ and 82 post-operation for sample dissection. For histological investigations five animals were sacrificed on days $1,3,7,14,28,42$ and 82 ; for the five animals designated for euthanasia on day 82 MR measurements were performed under anesthesia on the same seven time-points.

The investigation time-points were set in short intervals during the first month post-injury in order to closely follow bone bridge formation and changes in the microvessel system. The long-term time-points of 42 and 82 days were determined to follow further growth, physeal remodeling and to detect a potential disappearance of the bone bridge.

\section{MR measurements}

Magnetic resonance imaging of the morphology and DCE-MRI were performed on a clinical $3 \mathrm{~T}$ scanner with $38 \mathrm{mT} / \mathrm{m}$ gradient strength (Tim Trio, Siemens AG, Erlangen, Germany). For enhanced image quality, especially suited surface received coils with a diameter of $18 \mathrm{~mm}$ (Rapid Biomedical, Rimpar, Germany) were used. Using an arrangement as shown in Fig. 1 a minimum distance to the rat knees and thereby a maximized SNR could be maintained for the complete duration of the study. Animals were placed in prone position with their knees in the center of the coils. This arrangement was positioned in a semicircular animal bed (Rapid Biomedical) providing a stable and reproducible assembly. As mentioned above, the measurements were performed on days 1, 3, 7, 14, 28, 42 and 82.

Morphological changes of the investigated area were imaged with a high-resolution 3D FLASH sequence $\left(T_{\mathrm{E}}=7 \mathrm{~ms}, T_{\mathrm{R}}=100 \mathrm{~ms}\right.$, FA $=15$, scanning matrix $256 \times 256, \mathrm{FOV}=50 \mathrm{~mm}$, THK $=0.72 \mathrm{~mm}$ ) and coronal slice orientation related to the scanner's reference system. Image quality was documented calculating $\mathrm{SNR}=\mu_{\text {signal }} / \sigma_{\text {corr }}$, in which $\mu_{\text {signal }}$ is the average image intensity in a region of interest and $\sigma_{\text {corr }}$ is the standard deviation of a background region corrected for Rayleigh distribution. The corrected standard deviation is calculated $\sigma_{\text {corr }}=\sigma_{\text {Rayleigh }} / 0.655$ with $\sigma_{\text {Rayleigh }}$ being the standard
Fig. 1 Arrangement of the surface coils for all MR measurements. Left: bottom view, right: arrangement of coils in the animal bed
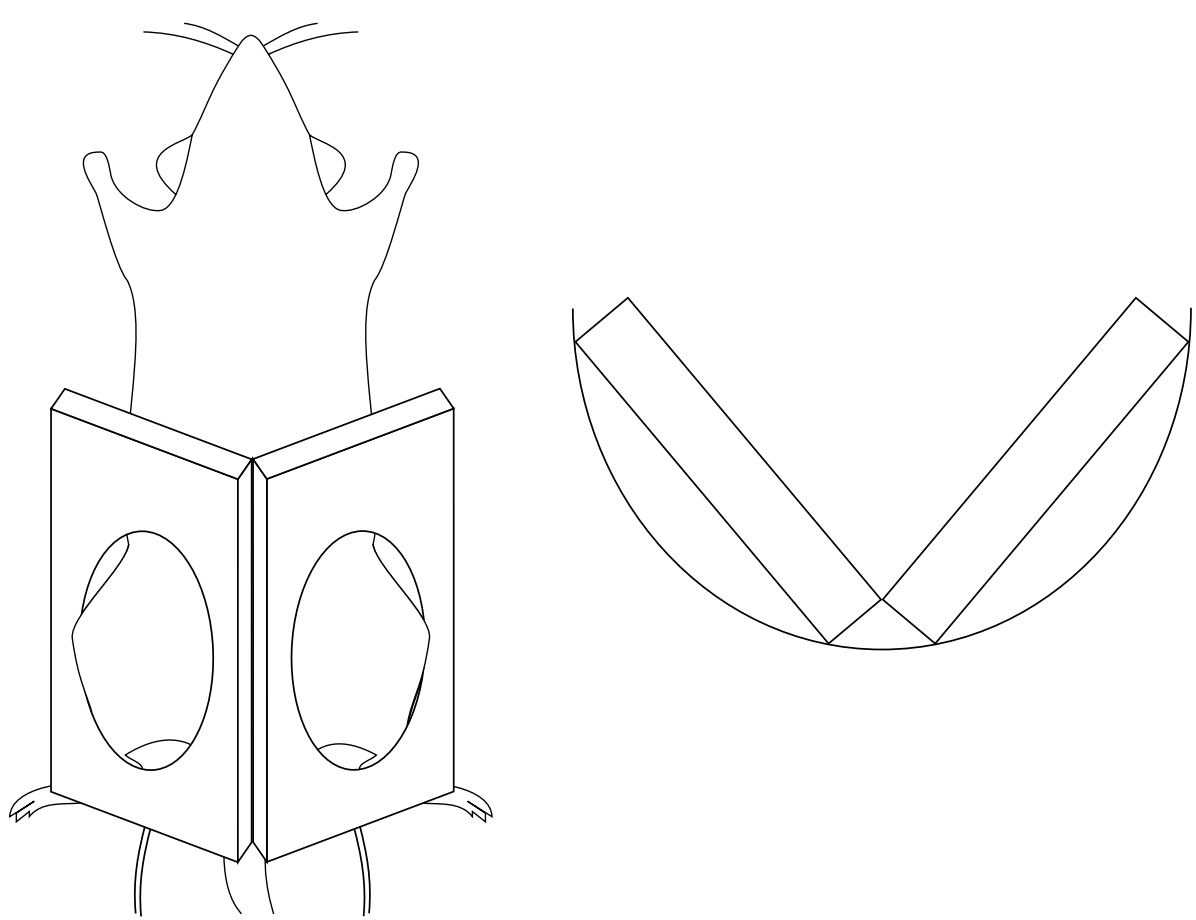
deviation measured in the image background region. Regions of interest for SNR determination were skeletal muscle and the physis.

The scans for DCE-MRI analysis comprised a single PDweighted 3D FLASH reference sequence $\left(T_{\mathrm{E}}=2.95 \mathrm{~ms}\right.$, $T_{R}=100 \mathrm{~ms}, \mathrm{FA}=5$ ) scan and a $T_{1}$-weighted dynamic 3D FLASH sequence $\left(T_{\mathrm{E}}=2.95 \mathrm{~ms}, T_{\mathrm{R}}=8.09 \mathrm{~ms}, \mathrm{FA}=30\right)$ with a temporal resolution of $\Delta t=13.32 \mathrm{~s}$. This combination follows the basic idea of the approach proposed by Hittmair et al. for 2D FLASH acquisitions [35]. Postoperatively a double-dose injection $(0.2 \mathrm{mmol} / \mathrm{kg})$ of Gadovist (Schering AG, Berlin, Germany) was manually administered intravenously in the tail vein for DCE-MRI. The administration started after the acquisition of three baseline images for the calculation of pre-contrast longitudinal relaxation time $T_{1}$; the entire protocol comprised 40 acquisitions. All scans for DCE-MRI were acquired in coronal orientation and featured a $205 \times 256$ matrix, a field of view of $50 \mathrm{~mm}$ and a slice thickness of $0.72 \mathrm{~mm}$.

\section{Reference region model}

Due to the absence of an adequate vessel providing an arterial input function in the FOV, blood supply related properties of the lesioned tissue were investigated by applying a reference region model [33] to the acquired DCE data. Maps of the longitudinal relaxation time $T_{1}$ were generated as proposed by Merwa et al. [36] according to Eq. (1)

$$
T_{1}(t)=-\frac{T_{R, \mathrm{DCE}}}{\ln \left(\frac{S_{\mathrm{REF}} \sin \left(\theta_{\mathrm{DCE}}\right)-S_{\mathrm{DCE}}(t) \sin \left(\theta_{\mathrm{REF}}\right)}{S_{\mathrm{REF}} \sin \left(\theta_{\mathrm{DCE}}\right)-S_{\mathrm{DCE}}(t) \sin \left(\theta_{\mathrm{REF}}\right) \cos \left(\theta_{\mathrm{DCE}}\right)}\right)},
$$

where $S$ is the signal intensity in the image, $\theta$ is the flip angle and the subscripts REF and DCE denote reference scan and dynamic scan, respectively. According to Yankeelov et al. [33] and using the notation suggested by Tofts et al. [24], the relaxation rate $R_{1} \equiv 1 / T_{1}$ of a tissue of interest (TOI) is related to the reference region by

$$
\begin{aligned}
R_{1, \mathrm{TOI}}(T)= & R \cdot\left(R_{1, \mathrm{RR}(t)}-R_{10, \mathrm{RR}}\right)+R \cdot\left[K^{\mathrm{trans}, \mathrm{RR}} / v_{\mathrm{e}, \mathrm{RR}}\right. \\
& \left.-K^{\mathrm{trans}, \mathrm{TOI}} / v_{\mathrm{e}, \mathrm{TOI}}\right] \times \int_{0}^{T}\left(R_{1, \mathrm{RR}}-R_{10, \mathrm{RR}}\right) \\
& \mathrm{e}^{\left(-K^{\mathrm{trans}, \mathrm{TOI}} / v_{\mathrm{e}, \mathrm{TOI}}\right)(T-t)} \mathrm{d} t+R_{10, \mathrm{TOI}} .
\end{aligned}
$$

In Eq. (2) $K^{\text {trans }}$ is the transfer coefficient, $v_{\mathrm{e}}$ is the extravascular extracellular volume, $R$ equals $K^{\text {trans,TOI }} / K^{\text {trans, } \mathrm{RR}}, R_{10}$ is the native relaxation rate in absence of a contrast agent and TOI and RR denote the tissue of interest and the reference region, respectively.

The reference region for all investigations was skeletal muscle close to the area of the physeal lesion, i.e. the tissue of interest, and the pharmacokinetic parameters of skeletal muscle were set to $K^{\text {trans,RR }}=0.045 \mathrm{~min}^{-1}$ and $v_{\mathrm{e}, \mathrm{RR}}=0.08$ [37-39]. For all measurements, the subjects' knees were located as close as possible to the center of the 3D slab to avoid different biases for the estimates of $R_{1}$ for RR and TOI due to $B_{1}$ inhomogeneities. The least mean squares routine for the parameter identification of $K^{\text {trans,TOI }}$ and $v_{\mathrm{e}, \mathrm{TOI}}$ was realized in MATLAB (TheMathWorks Inc., Natick, MA, USA).

\section{Histology}

To obtain information on vascularisation complementary to the results of the analysis of the pharmacokinetic parameters, immunohistochemical analysis was performed for samples of the proximal epiphysis, physis and metaphysis of the tibial bone on the same days for a second cohort of animals.

Histological samples were fixed in methanol $(100 \%)$ for $24 \mathrm{~h}$ and subsequently subjected to decalcification using Ethylenediamine Tetraacetic Acid ( $\mathrm{pH}$ 7.0) for two weeks. Following a further fixation step in methanol (100\%) for $12 \mathrm{~h}$ the samples were washed in PBS-sucrose 5\% solution, covered with tissue freezing medium (Tissue Tek O.C.T. Compound, Sakura Finetek Europe B.V., The Netherlands) and cut into slices of $7 \mathrm{~mm}$ thickness.

To visualize angiogenesis of the physeal defect a collagen IV staining was used, as collagen IV represents a protein of the basal membrane of vessels, staining vasculature in a red/brown colour.

\section{Quantitative real-time reverse transcription polymerase chain reaction}

Additionally to MR measurements and histological analysis, factors related to vascular growth and oxygenation were determined using quantitative real-time reverse transcription polymerase chain reaction analyses. The qRTPCR analyses were performed for vascular endothelial growth factor a (VEGFa), which is a key parameter of angiogenesis [40] and hypoxia-inducible factor 1a (Hif1a) as a reporter for hypoxia.

For the analysis, physeal samples were dissected from the epiphysis and metaphysis under the microscope before storage in liquid nitrogen. The samples were homogenized using a tissue homogenizer and RNA was extracted using TRIZOL (Gibco BRL). Total RNA samples $(2.5 \mathrm{mg})$ were reverse transcribed at $42{ }^{\circ} \mathrm{C}$ for 15 min using random hexamer priming. To establish quantitative expression profiles for VEGFa and Hif1a, commercially available, pre-optimized real time-PCR assays (Assay On Demand, Applied Biosystems) were used. Three reference genes (internal controls) were used for relative quantification. Normfinder [41] software was used to identify the optimal reference gene for internal normalization from a set of house-keeping 
genes. Triplicates were averaged and data was normalized to the house keeping gene $\beta$-actin of day 0 .

\section{Statistical analysis}

Statistical analysis was performed in SPSS 22 (SPSS Chicago, IL). Differences between results were tested for significance applying a post hoc Bonferroni test and a $p$ value threshold of 0.05 for statistical significance; a p-value below 0.01 was considered highly significant.

\section{Results}

Figure 2 shows the high resolution and the excellent contrast of the morphological images acquired with the setting described above. The scans show the changes of the lesioned area of the physis (marked by arrows) of one representative animal for all 7 time points of the study and document the formation of bone bridges: On days 1 and 3 after the drill procedure, the drill mark causing physeal interruption is clearly visible. On day 7 the physeal interruption caused by the drill mark disappears leaving a homogenous high signal physis. A first low-signal bone bridge can be seen as early as on day 14 and progressively becomes more defined as a larger low-signal interruption within a high signal physis. These interruptions did not dissolve until the end of the study. SNR values showed an observable decrease after two weeks: skeletal muscle exhibited an average SNR of 82 in the first 14 days and 76 afterwards. For the physis, the SNR dropped from 72 to 36 on average.

The analysis of the reference regions of all days and subjects yielded a relaxation rate of $0.82 \pm 0.17 \mathrm{~s}^{-1}$. The results for the transfer coefficient and the extravascular extracellular volume provided by the reference region model are shown in Fig. 3: on day 1 post-operation $K^{\text {trans }}$ is at a minimum and $v_{\mathrm{e}}$ is notably high; however, with strong variations. On day 3 a non-significant increase of the transfer coefficient accompanied by a decrease in $v_{\mathrm{e}}$ is evident. The value of $K^{\text {trans }}$ on day 7 is significantly increased compared to day 1 and reaches its maximum on day 42 postoperation (highly significant different from day 14) with a concomitant increase of the extravascular extracellular space. Between the results of days 7, 14 and 28 no statistically significant differences exist. The value for the transfer coefficient decreased significantly from day 42 to day 82 accompanied by a decrease of $v_{\mathrm{e}}$. For the entire study the results for $v_{\mathrm{e}}$ did not show significant differences between measurement days.

Concerning factors of vascular growth, a first visible but non-significant increase occurs on day 1 for VEGFa as an immediate reaction to the operation (Fig. 4). The only significant difference for VEGFa can be observed on day 7 post-lesion which shows increased values compared to the baseline (day 0) and days 14 and 82 . The expression of Hif1a increases from day 1 on with maximum values on day 3 yielding a highly significant increase compared to days 0,14 and 82 .
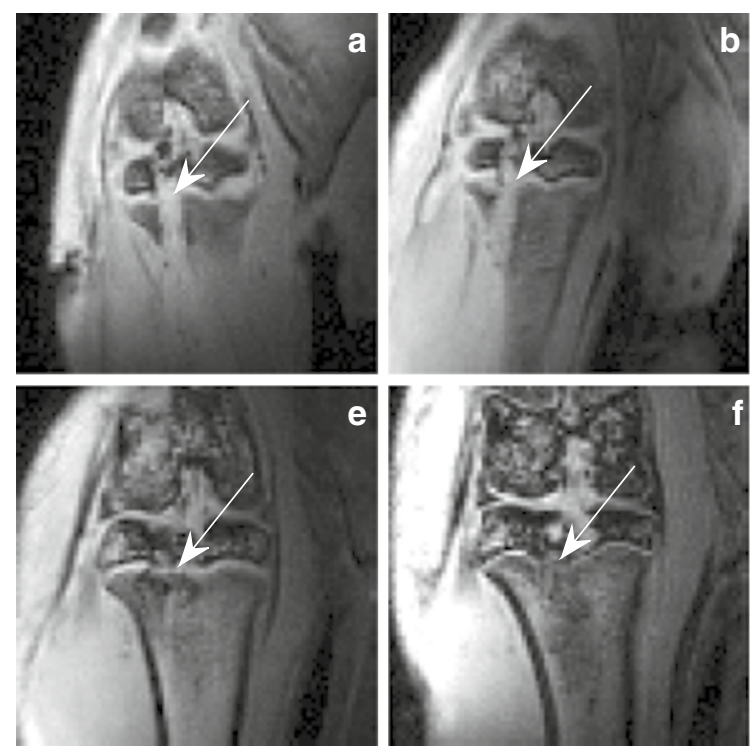

Fig. 2 High resolution, non-contrast-enhanced MR Images of the lesioned area (arrow) in the tibial physis of one subject on days $\mathbf{a} 1, \mathbf{b}$ 3 , c 7 , d 14 , e 28 , f 42 and $\mathbf{g} 82$ post-lesion (images were brightened
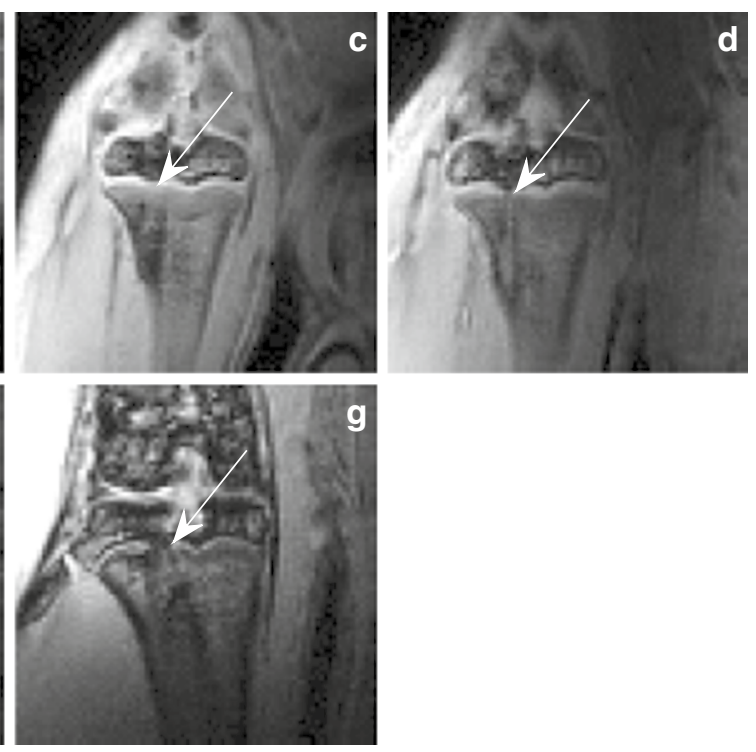

9

for a better view of the anatomy). The formerly disrupted area looks closed on day 7 but on day 28 it is clearly disrupted again 
Fig. 3 Results for $\mathbf{a} K^{\text {trans }}$ and b $v_{e}(n=5$ for each day). Horizontal bars indicate the median, the 25 th and 75 th percentiles. The values for the transfer coefficient start to increase after 3 days and reach their maximum on day 42 post-operation. The volume of the extravascular extracellular space is increased on days 1 and 42 but does not show statistically significant changes. Statistical changes are marked with $* p<0.05$ and $* * p<0.01$, respectively

Fig. 4 Quantitative real time reverse transcription PCR results for $\mathbf{a}$ VEGFa and $\mathbf{b}$ Hifla ( $n=6$ for each day). Horizontal bars indicate the median, the 25th and 75th percentiles, minor outliers are displayed with a circle; major outliers with a star. Maximum vascular growth occurs on day 7 (VEGFa), forgone by maximum hypoxia on day 3 (Hifla). Statistical changes are marked with $* p<0.05$ and $* * p<0.01$, respectively
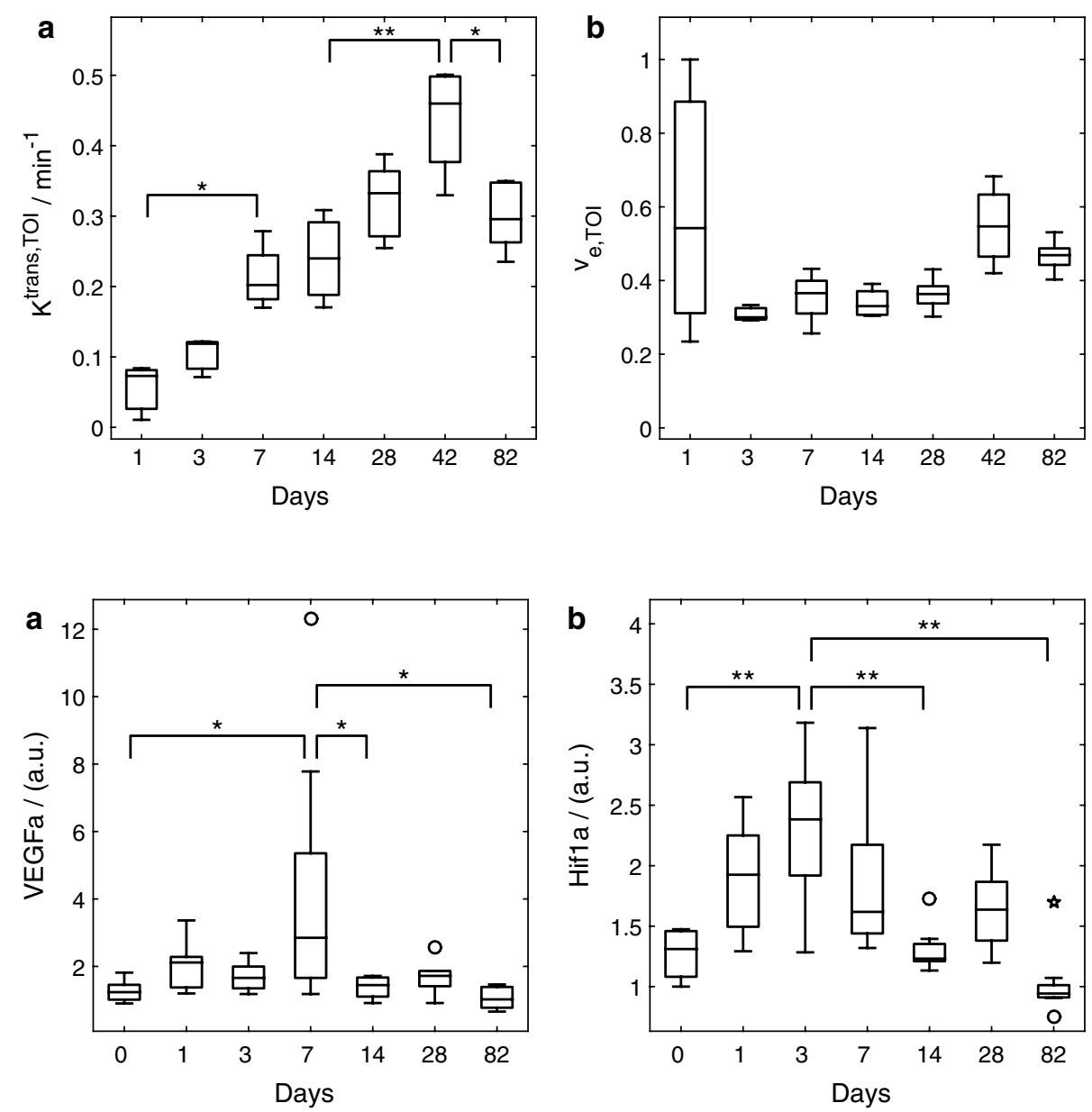

Finally, histological investigations additionally showed the development of microvasculature in the physeal defect. Figure 5 shows collagen IV-stained histologic slices of days $1,3,7,14,28,42$ and 82 of the physeal lesion. On day 1 the staining showed no vascularisation. On days 3 and 7 post-operation the staining clearly displays the presence of capillaries (marked by arrowheads). After two weeks, first bony trabeculae (collagen IV-negative and marked by arrows) are observed within the physeal defect site. At this time-point collagen IV-positive vessels appear to be more structured compared to days 3 and 7 . On day 42 the defective area is filled with lamellar bone fragments, which are surrounded by vessels. On day 82 post-operation the number of collagen IV-positive vessels appear reduced compared to day 42 .

\section{Discussion}

In order to detect bone bridge formation at an early stage using a minimally invasive method a reference region model was applied to DCE-MRI data of injured physes to analyze the changes of the microvessel system of this tissue. Furthermore, the changes of the pharmacokinetic parameter $K^{\text {trans }}$ over time were compared to qRT-PCR data and results of immunohistochemical analyses and the development of bone bridges was observed using high-resolution MRI.

The performed MRI studies show significant and clear time dependent changes of the assessed model parameter $K^{\text {trans }}$. This supports the hypothesis that changes of the blood supply are a potential mechanism associated with bone bridge formation of injured physes and permits a detection of the formation even before it becomes visible in MR imaging data.

Although this study was performed on a whole-body system, high quality morphological images could be obtained by using a dedicated receiver coil and by optimizing the parameters of the 3D gradient echo sequence. The used setting provided images with a voxel size of $0.19 \mathrm{~mm} \times 0.19 \mathrm{~mm} \times 0.72 \mathrm{~mm}$, which allowed for an identification of the relevant image details. For the second half of the study the image quality was noticeably decreased. This is owed to the growth of the animals causing an increase in the distance between the surface coils and the region of interest, which consequently lead to a 
decrease of the SNR of the acquired data. However, the sharp decrease for the SNR of the physis after 2 weeks is also caused by a diminished signal intensity of the region of interest due to the animals' age. This is also reflected by the much less pronounced decrease in SNR for skeletal muscle. The images did not suffer from susceptibility artifacts; however, for higher field strengths this may be the case, as reported by Taha et al. [42], who found a RARE approach to be superior to FLASH at $9.4 \mathrm{~T}$.

The high quality of the morphological scans provided a good insight into physeal bone bridge formations throughout the whole study. As was to be expected, the lesioned area can be clearly identified shortly after the application of the drilling procedure and the refillment of the injury was largely completed on day 7 post-operation. By only investigating image data for this day, this refillment could be accredited to healing processes closing the physeal lesion. While a remaining damage of the tibia was still visible, the formerly lesioned area of the physis was homogeneously closed and the isointensity of the filled lesion compared to the surrounding physis suggests a refillment with cartilaginous tissue.

First changes in the investigated area become slightly visible in the MR images on day 14 but without additional information these changes cannot be positively assigned to a bone bridge formation. However, the discontinuity of the physeal area on day 28 is a strong indication for bony activity. This new interruption of the physis occurred in all examined subjects indicating a strong connection between injuries across the physis and bone bridge-induced defective healing, as suggested in the literature [13, 15, 17, 34]. The onset of bone bridge formation is in good accordance with findings of a recent study investigating the efficacy of MRI for the detection of changes in bone morphology [42].

The specific requirements of this animal study lead to a moderate temporal resolution of the dynamic scan. This and the lack of a feeding artery in the investigated area required the application of an RRM to acquire pharmacokinetic parameters. The reference region was skeletal muscle and the average relaxation rate of the reference regions of all days and subjects is in good agreement with literature values $[43,44]$. The analysis of the pharmacokinetic parameter $K^{\text {trans }}$ revealed changes in the investigated area, which were not visible in imaging data. These changes can be attributed to the underlying mechanisms of the formation of bone bridges, which did not become visible in the morphological images before day 14 and could not be clearly identified before day 28 post-operation. On days 1 and 3 post operation the lesion is mostly filled with blood, thus $K^{\text {trans }}$ cannot be reasonably determined. Especially on the first measurement day the development of blood vessels is expected to be still very low, which hinders a meaningful parameter identification. This, for instance, is reflected in the quite strong variance of the values for $v_{\mathrm{e}}$. However, from day 3 onwards the fitting routines provide robust, i.e. less scattering, results and the increase of $K^{\text {trans }}$ suggests a proliferation of microvessels.

While it is not completely clear whether such an establishment of a microvessel structure triggers a defective healing and thereby hinders a restitutio ad integrum, it can be considered as a first sign for the formation of bone bridges. This is a remarkable finding, since prior to this study date neither histologic analysis nor MR image data could unambiguously detect newly formed bone tissue. Histology could confirm the presence of bone fragments in the lesioned area [13] but it is still disputed if these bone fragments within a fracture site lead to the formation of bone bridges, as according to Xian et al. [17], an irrigation of the drill track had no measurable effect on the development of osseous matter. Therefore, the determination of $K^{\text {trans }}$ allows for an earlier detection of bone bridge formation.

After day 3 post-operation the transfer coefficient indicates a steady increase. The maximum value of $K^{\text {trans }}$ on day 42 reflects the blood supply of bone bridges, which could be clearly detected in the morphological images at that time.

The volume of the extravascular extracellular space did not provide additional information. The decrease at the beginning of the study could be interpreted as a sign for increased blood supply; however, it was reported that the RR estimate of $v_{\mathrm{e}}$ and the estimate produced by the standard method with known AIF are not correlated [45]. Therefore, and since the changes of this parameter were only statistically non-significant the course of this quantity was not investigated further.

The analysis of collagen IV-stained histology data confirmed the presence of vascularity from day 3 onwards, which coincided with an increase of $K^{\text {trans }}$. After two weeks the stained vessels appear less scattered but greater in size and first formations of bone bridges can be detected. This is followed by another increase of $K^{\text {trans }}$ until day 42 followed by a decrease between days 42 and 82 , which coincides with a seemingly reduced staining. The voxel based integrative parameter $K^{\text {trans }}$ show a smoother development with less variation in comparison to parameter from histological samples.

The increased expression of VEGFa on day 7 is preceded by a rise in the expression of Hifla with its maximum on day 3. VEGF expression has been reported to be induced by hypoxia [46]. Furthermore, the heterodimeric basic helix-loop-helix protein Hif1 has been attributed to directly activate VEGF transcription in hypoxic cells, implicating Hifla to play a pivotal role within this cascade [47, 48]. Although, VEGF and Hif1a were not measured 

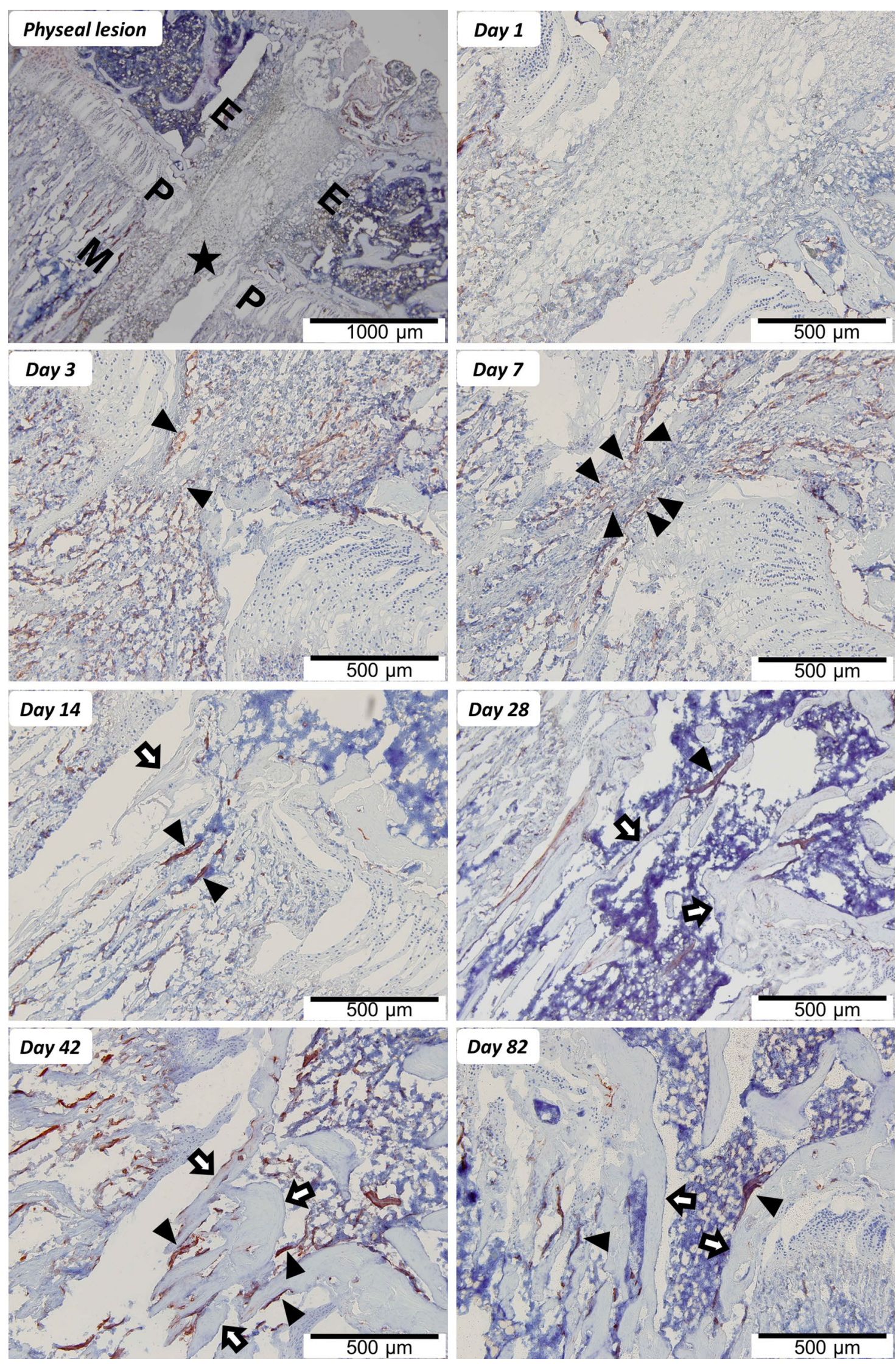
4Fig. 5 Immunohistochemical expression pattern of collagen IV demonstrating vascular ingrowth preceding bone bridge formation at the physeal lesion site. "Physeal lesion" shows an overview of the physeal lesion (filled star lesion, $P$ physis, $E$ epiphysis, $M$ metaphysis of the tibial head). In the remaining images collagen IV-positive vascularization, stained red/brown, is highlighted by arrowheads (filled pointer), while arrows (white arrow) mark bone fragments which eventually form a bone bridge. The physeal lesion is postoperatively filled by hematoma without obvious vascular demarcation (day 1). Day 3 shows first capillaries ingrowing from surrounding hematoma into the physeal lesion site (indicated by arrowheads). The maximum number of capillaries pervading the physeal lesion are seen on day 7 . Day 14 shows bony trabeculae traversing the physeal defect, maturing to a bone bridge connecting the epiphysis with the metaphysis by day 28 (marked by arrows). By day 42 the bone bridge consists of lamellar bone and is accompanied by distinct vessels, which decline with further maturation of the bridge (day 82)

on protein levels in our study, we suspect that, based on our qRT-PCR results, the changes of the microvessel system and the increased values of $K^{\text {trans }}$ occurring on day 7 were most probably triggered by hypoxia within the physeal lesion site. The scattered VEGFa expression observed primarily on day 7 is presumably associated with the relative small sample size, where a short increase of expression towards the randomly selected day 7 may be hard to detect exactly. The statistical outlier of day 7 , however, may be due to a contaminated sample by surrounding tissue.

Comparing the results of MR imaging and the pharmacokinetic analyses to the complementary data it can be stated that the increase in $K^{\text {trans }}$ corresponds to a filling of the physeal injury with vasculature. The data also shows that the non-significant increase of $K^{\text {trans }}$ between days 7 and 14 coincides with a return of VEGFa expression to its initial value on day 14 . However, it is also notable that qRT-PCR results reveal a rather pronounced variance in the data while the pharmacokinetic analysis provides a clearly identifiable trend. The results suggest that the combination of high-resolution MRI with a pharmacokinetic analysis allows to identify bone bridge formation at an early stage, while being only minimally invasive.

Currently, Gadolinium-based contrast agents are not generally used for the detection of physeal bone bridges; however, contrast-enhanced measurements are already applied for different disorders, such as the Legg-CalvéPerthes disease [49]. Therefore, we expect our method to be generally applicable to children, which would provide a quantitative measure for changes in the micro-vessel system associated with the formation of bone bridges at an early stage. The detection of changes in $K^{\text {trans }}$ was at least 7 days earlier than the detection of first morphological signs for bone bridges. This time period may translate from the specific animal model to a duration between 6 and 19 months for humans [50, 51], which can be particularly important in cases with concealed physeal bridging. Clinically, many physeal bone bridges may not become evident until months or years have passed and growth disturbances have developed [1]. This highlights the necessity for early recognition of physeal bone bridge formation, which subsequently aids surgical management in order to restore potential growth to the bone.

\section{Conclusion}

In this study, we used DCE-MRI on a clinical system to assess parameters related to blood supply in an animal model in order to investigate the possibility to detect the formation of bone bridges using a minimally invasive method. We conclude that a transphyseal lesion is associated with increased values for $K^{\text {trans }}$ which could be observed from day 3 onwards using DCE-MRI. The increase of the transfer coefficient coincides with a presence of vascularity, which appears to trigger the consecutive bone bridge formation and thereby may hinder a restitutio ad integrum of the physeal defect.

MR measurements did not only document the formation of bone bridges but additionally provided quantitative information on tissue development and microvessel proliferation in the lesioned area. The analysis of the DCE data enabled an indirect detection prior to visibility in morphology images, which may help in preventing growth disturbances in children by allowing therapeutic intervention early in course. This finding, however, still needs verification in a human study.

Acknowledgements Open access funding provided by Austrian Science Fund (FWF). This work was funded by the Austrian Science Fund under Grant SFB F3209-18 and by the AO Research Fund of the AO Foundation, Project S-06-96 W.

Authors' Contribution The medical part of the study was defined by EA and AMW, RS was responsible for MRI and pharmacokinetic modelling. Protocols for MR measurements were developed by $\mathrm{CD}$ and RS, EA and AMW supervised animal surgery, qRT-PCR and histological investigations. Neumayer implemented, tested and applied the pharmacokinetic model and performed pre-processing for quantification. Data analysis was made by BN, CD, ES and RS. BN, CD, EA and RAL were involved in MR data collection, EW conducted the histological experiments and performed the analyses. The manuscript was mainly written by BN; EA, ES and RS contributed to specific parts. The manuscript has been proof-read and approved by all named authors.

\section{Compliance with ethical standards}

Conflict of interest The authors declare that they have no conflict of interest.

Ethical statement All applicable international, national, and/or institutional guidelines for the care and use of animals were followed. All 
procedures performed in studies involving animals were in accordance with the ethical standards of the Austrian Ministry of Science and Research.

Open Access This article is distributed under the terms of the Creative Commons Attribution 4.0 International License (http://creativecommons.org/licenses/by/4.0/), which permits unrestricted use, distribution, and reproduction in any medium, provided you give appropriate credit to the original author(s) and the source, provide a link to the Creative Commons license, and indicate if changes were made.

\section{References}

1. Wang DC, Deeney V, Roach JW, Shah AJ (2015) Imaging of physeal bars in children. Pediatr Radiol 45(9):1403-1412

2. Aitken AP, Magill HK (1952) Fractures involving the distal femoral epiphyseal cartilage. J Bone Joint Surg Am 34A:96-108

3. Boelitz R, Dallek M, Meenen NM, Jungbluth KH (1994) Die Reaktion der Epiphysenfuge auf fugenkreuzende Bohrdrahtosteosynthesen. Unfallchirurgie 20:131-137

4. Ecklund K, Jaramillo D (2002) Patterns of premature physeal arrest: MR imaging of 111 children. AJR Am J Roentgenol 178(4):967-972

5. Escott BG, Kelley SP (2012) Management of traumatic physeal growth arrest. Orthop Trauma 26:200-211

6. von Laer L (1994) Natural course following fractures during the growth years. Orthopade 23(3):211-219

7. Weinberg A, Tscherne H (2006) Tscherne Unfallchirurgie: Unfallchirurgie im Kindesalter-Teil 1: Allgemeiner Teil, Kopf, Obere Extremität. Springer, Berlin

8. Ecklund K, Jaramillo D (2001) Imaging of growth disturbance in children. Radiol Clin North Am 39(4):823-841

9. Sailhan F, Chotel F, Guibal AL, Gollogly S, Adam P, Bérard J, Guibaud L (2004) Three-dimensional MR imaging in the assessment of physeal growth arrest. Eur Radiol 14(9):1600-1608

10. Shukrimi AB, Afizah MH, Schmitt JF, Hui JH (2013) Mesenchymal stem cell therapy for injured growth plate. Front Biosci S5:774-785

11. Tomaszewski R, Bohosiewicz J, Gap A, Bursig H, Wysocka A (2014) Autogenous cultured growth plate chondrocyte transplantation in the treatment of physeal injury in rabbits. Bone Joint Res 3:310-316

12. Kennon JC, Ganey TM, Gaston RG, Ogden JA (2013) Continued growth after limited physeal bridging. J Pediatr Orthoped 33:857-861

13. Fischerauer E, Heidari N, Neumayer B, Deutsch A, Weinberg AM (2011) The spatial and temporal expression of VEGF and its receptors 1 and 2 in post-traumatic bone bridge formation of the growth plate. J Mol Histol 42:513-522

14. Garcés GL, Mugica-Garay I, Coviella NL-G, Guerado E (1994) Growth-plate modifications after drilling. J Pediatr Orthoped $14: 225-228$

15. Jaramillo D, Shapiro F, Hoffer FA et al (1990) Posttraumatic growth- plate abnormalities: MR imaging of bony-bridge formation in rabbits. Radiology 175:767-773

16. Jaramillo D, Laor T, Zaleske DJ (1993) Indirect trauma to the growth plate: results of MR imaging after epiphyseal and metaphyseal injury in rabbits. Radiology 187:171-178

17. Xian CJ, Zhou FH, McCarty RC, Foster BK (2004) Intramembranous ossification mechanism for bone bridge formation at the growth plate cartilage injury site. J Orthop Res 22:417-426
18. Koff MF, Chong LR, Virtue P, Ying L, Gholve PA, Rodeo SA, Widmann RF, Potter HG (2010) Correlation of magnetic resonance imaging and histologic examination of physeal bars in a rabbit model. J Pediatr Orthop 30:928-935

19. Shapiro F (1982) Epiphyseal growth plate fracture-separations: a pathophysiologic approach. Orthopedics 5:720-736

20. Widni EE, Hausbrandt P, Kraitsy K, Deutsch A, Höllwarth ME, Weinberg AM (2010) Physeal bone bridge formation after transphyseal lesion relapses endochondral ossification. In: Proceedings of the 77 th AAOS annual meeting, New Orleans, $p$ 143

21. Padhani AR (2002) Dynamic contrast-enhanced MRI in clinical oncology: current status and future directions. J Magn Reson Imaging 16:407-422

22. Neeman M (2000) Preclinical MRI experience in imaging angiogenesis. Cancer Metastasis Rev 19:39-43

23. Gillies RJ, Bhujwalla ZM, Evelhoch J, Garwood M, Neema M, Robinson SP, Sotak CH, Van Der Sanden B (2000) Applications of magnetic resonance in model systems: tumor biology and physiology. Neoplasia 2:139-151

24. Tofts PS, Brix G, Buckley DL, Evelhoch JL, Henderson E, Knopp MV, Larsson HB, Lee T-Y, Mayr NA, Parker GJ, Port RE, Taylor J, Weisskoff RM (1999) Estimating kinetic parameters from dynamic contrast-enhanced T1-weighted MRI of a diffusable tracer: standardized quantities and symbols. J Magn Reson Imaging 10:223-232

25. Ashton E, Raunig D, Ng C, Kelcz F, McShane T, Evelhoch J (2008) Scan-rescan variability in perfusion assessment of tumors in MRI using both model and data-derived arterial input functions. J Magn Reson Imaging 28:791-796

26. Jackson A, Buckley DL, Parker GJM (2006) Dynamic contrastenhanced magnetic resonance imaging in oncology. Springer, Berlin

27. Yankeelov TE, Gore JC (2009) Dynamic contrast enhanced magnetic resonance imaging in oncology: theory, data acquisition, analysis, and examples. Curr Med Imaging Rev 3:91-107

28. Port RE, Knopp MV, Brix G (2001) Dynamic contrast-enhanced MRI using Gd-DTPA: interindividual variability of the arterial input function and consequences for the assessment of kinetics in tumors. Magn Reson Med 45:1030-1038

29. Wang Y, Huang W, Panicek DM, Schwartz LH, Koutcher JA (2008) Feasibility of using limited-population-based arterial input function for pharmacokinetic modeling of osteosarcoma dynamic contrast-enhanced MRI data. Magn Reson Med 59:1183-1189

30. Li X, Welch EB, Arlinghaus LR, Chakravarthy AB, Xu L, Farley J, Loveless ME, Mayer IA, Kelley MC, Meszoely IM et al (2011) A novel AIF tracking method and comparison of DCEMRI parameters using individual and population-based AIFs in human breast cancer. Phys Med Biol 56:5753-5769

31. McGrath DM, Bradley DP, Tessier JL, Lacey T, Taylor CJ, Parker GJ (2009) Comparison of model-based arterial input functions for dynamic contrast-enhanced MRI in tumor bearing rats. Magn Reson Med 61:1173-1184

32. Kovar DA, Lewis M, Karczmar GS (1998) A new method for imaging perfusion and contrast extraction fraction: input functions derived from reference tissues. J Magn Reson Imaging 8:1126-1134

33. Yankeelov TE, Luci JJ, Lepage M, Li R, Debusk L, Lin PC, Price RR, Gore JC (2005) Quantitative pharmacokinetic analysis of DCE-MRI data without an arterial input function: a reference region model. Magn Reson Imaging 23:519-529

34. Lee MA, Nissen TP, Otsuka NY (2000) Utilization of a murine model to investigate the molecular process of transphyseal bone formation. J Pediatr Orthop 20:802-806

35. Hittmair K, Gomiscek G, Langenberger K, Recht M, Imhof H, Kramer J (1994) Method for the quantitative assessment of 
contrast agent uptake in dynamic contrast-enhanced MRI. Magn Reson Med 31:567-571

36. Merwa R, Reishofer G, Feiweier T, Kapp K, Ebner F, Stollberger $R$ (2009) Impact of B1 inhomogeneities on AIF selection in DCE-MRI at 3 Tesla. In: Proceedings of the 17th scientific meeting, international society for magnetic resonance in medicine, Honolulu, p 4661

37. Yankeelov TE, Luci JJ, DeBusk LM, Lin PC, Gore JC (2008) Incorporating the effects of transcytolemmal water exchange in a reference region model for DCE-MRI analysis: theory, simulations, and experimental results. Magn Reson Med 59:326-335

38. Landis CS, Li X, Telang FW, Molina PE, Palyka I, Vetek G, Springer CS (1999) Equilibrium transcytolemmal waterexchange kinetics in skeletal muscle in vivo. Magn Reson Med 42:467-478

39. Buckley DL, Kershaw LE, Stanisz GJ (2008) Cellular-interstitial water exchange and its effect on the determination of contrast agent concentration in vivo: dynamic contrast-enhanced MRI of human internal obturator muscle. Magn Reson Med 60:1011-1019

40. Ferrara N, Gerber H-P, LeCouter J (2003) The biology of VEGF and its receptors. Nat Med 9:669-676

41. Andersen CL, Jensen JL, Ørntoft TF (2004) Normalization of real-time quantitative reverse transcription-PCR data: a modelbased variance estimation approach to identify genes suited for normalization, applied to bladder and colon cancer data sets. Cancer Res 64:5245-5250

42. Taha MA, Manske SL, Kristensen E, Taiani JT, Krawetz R, Wu Y, Ponjevic D, Matyas JR, Boyd SK, Rancourt DE, Dunn JF (2013) Assessment of the efficacy of MRI for detection of changes in bone morphology in a mouse model of bone injury. $\mathrm{J}$ Magn Reson Imaging 38:231-237
43. Donahue KM, Weisskoff RM, Parmelee DJ, Callahan RJ, Wilkinson RA, Mandeville JB, Rosen BR (1995) Dynamic GdDTPA enhanced MRI measurement of tissue cell volume fraction. Magn Reson Med 34:423-432

44. Yankeelov TE, Rooney WD, Li X, Springer CS (2003) Variation of the relaxographic "shutter-speed" for transcytolemmal water exchange affects the CR bolus-tracking curve shape. Magn Reson Med 50:1151-1169

45. Yankeelov TE, Cron GO, Addison CL, Wallace JC, Wilkins RC, Pappas BA, Santyr GE, Gore JC (2007) Comparison of a reference region model with direct measurement of an AIF in the analysis of DCE-MRI data. Magn Reson Med 57:353-361

46. Shweiki D, Itin A, Soffer D, Keshet E (1992) Vascular endothelial growth factor induced by hypoxia may mediate hypoxia-initiated angiogenesis. Nature 359:843-845

47. Forsythe JA, Jiang BH, Iyer NV, Agani F, Leung SW, Koos RD, Semenza GL (1996) Activation of vascular endothelial growth factor gene transcription by hypoxia-inducible factor 1 . Mol Cell Biol 16(9):4604-4613

48. Weidemann A, Johnson RS (2008) Biology of HIF-1alpha. Cell Death Differ 15(4):621-627

49. Kim HKW, Wiesman KD, Kulkarni V, Burgess J, Chen E, Brabham C, Ikram H, Du J, Lu A, Kulkarni AV, Dempsey M, Herring JA (2014) Perfusion MRI in early stage of Legg-Calvé-Perthes disease to predict lateral pillar involvement. J Bone Joint Surg Am 96(14):1152-1160

50. Quinn R (2005) Comparing rat's to human's age: how old is my rat in people years? Nutrition 21:775-777

51. Andreollo NA, dos Santos EF, Araújo MR, Lopes LR (2012) Rat's age versus human's age: what is the relationship? Arq Bras Cir Dig 25:49-51 